\title{
Sustainable water purification using biomass
}

Nanoscale cellulose materials obtained from the chemical treatment of biomass are very effective agents for the
removal of toxic species from water, including heavy metal ions. Professor Benjamin $S$. Hsiao and his collaborators at Stony Brook University

inexpensive and environmentally friendly approach to preparing nanostructured cellulose for water purification, based on a nitro-oxidation reaction carried out on biomasses of diverse origins. In addition to providing cellulose with a superior affinity for dissolved toxicions, this process yields nitrogen-rich salts recovered and used as fertilizers. he ability to remove pollutants
quickly and efficiently at low cost
is a basic requisite for the human utilisation of water in a large variety of environments and situations. For instance, which is extensively used in electronic in many developing countries, clean water circuits, batteries, solar cells, paints remains a rare and precious commodity, and pigments, and can enter water since the available (and often very limited) sources through industrial waste and sources of water frequently contain ran-offs. Consumption of water or food incompatible with human consumption. to severe gastrointestinl intation In industrialised societies, metal pollutants potentially to death. Increasing tevels also pose a severe threat to health of cadmium contamination have been and the environment.

Dr Hsiao's team has been at the forefront of research on the chemical modification of nanostructured cellulose (nanocellulose) for water purification and they have recently demonstrated a simple, innovative, and environmentally friendly approach to exploit nanocellulose from virtually ubiquitous and low-cost natural

HEAVY METAL

\section{CONTAMINANTS IN WATER}

Heavy metal ions are among the most in modern societies. For instance, lead ions are powerful neurotoxins and they constitute the most prevalent form of heavy metal water pollution on a global scale. Over a hundred thousand deaths attributed to lead poisoning have been reported in 2016. Lead poisoning has also been linked to the appearance of defects at birth and to cancer. Lead is commonly used in the infrastructures for water

transportation and supply around the world, and the amount of metal dissolved in drinking water increases with time due to the progressive corrosion of the infrastructure. Furthermore, the recent practice to add chloramine for disinfection in water treatment facilities has led to in drinking water, because of the reaction

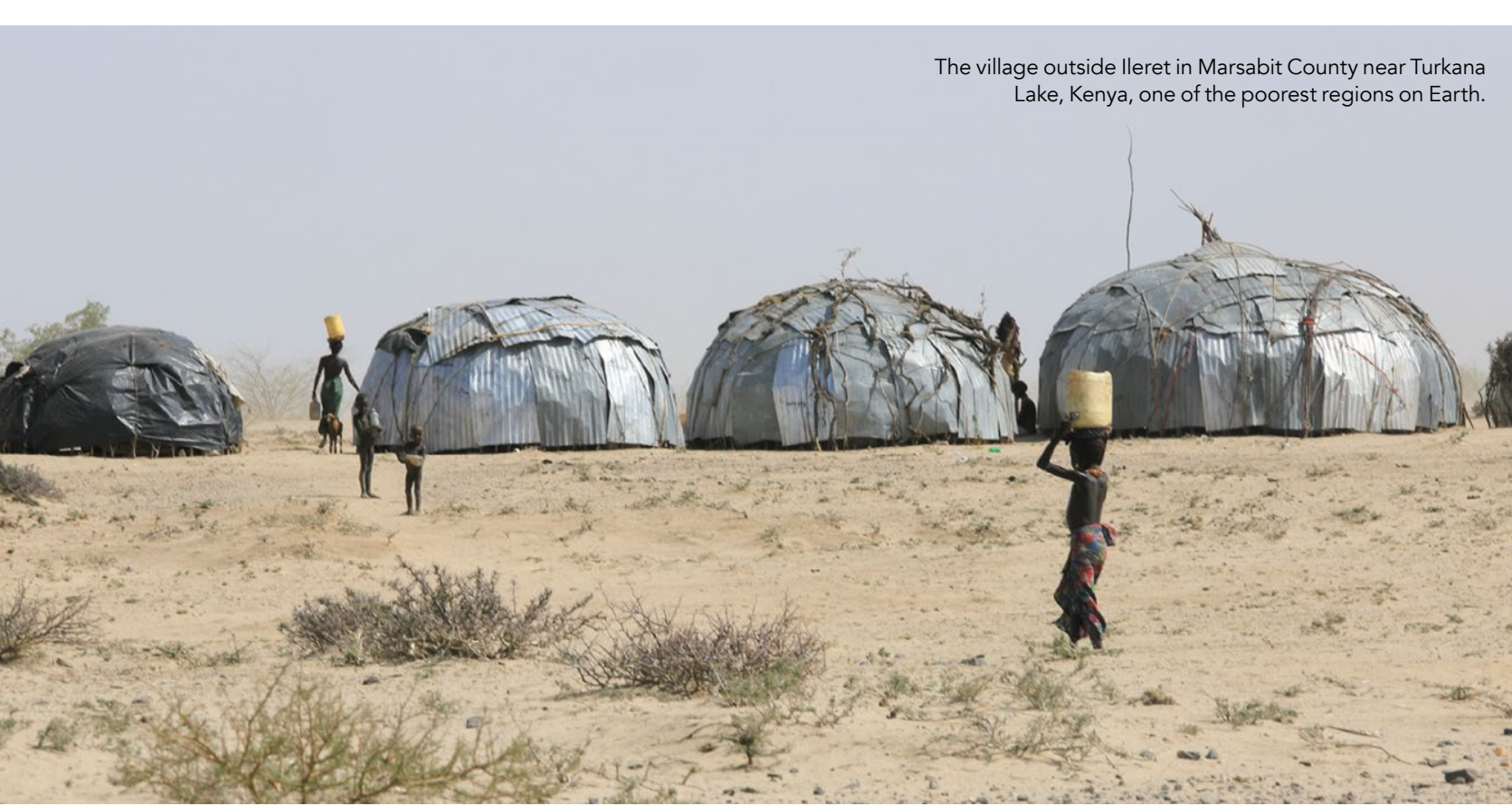

of materials for water purification, and low environmental impact, as it can be extracted from any biomass such as trees, plants and weeds.

\section{CARBOXYCELLULOSE:} A FUNCTIONAL MATERIAL FOR WATER PURIFICATION Cellulose is the most abundant organic polymer on Earth, and it is a primary component of the cell walls in plan t. It is composed of long chains of D-glucose units, connected by bridging oxygen atoms. It is abundant in natural flbres (for instance, its content in cotto is roughly $90 \%$ ) and in wood ( $40-50 \%$ ) Is rovisy $90 \%$ and in wood (40-50\%) including paper, cellophane and rayon. Cellulose derivatives obtained by chemical treatment of raw cellulose can also bind efficiently with metal ions in water.

The work of Dr Hsiao has focused specifically on carboxycellulose, which is composed of cellulose chains that have been chemically modified to include carboxylate groups

(-COO) in their structure. Nanostrcutured carboxycellulose has two key features, which make it highly attractive as an ion-binding material. First, its nanoscale structure originates from the existence in the cell walls of (cellulose microfibrils) ther than from the riombin dissolved cellulose chains. The production of nanostructured carboxycellulose, therefore, does not necessarily require energy-intensive processes Second the chemical modification of the cellulose carboxymethylation, phosphorylation,

\section{NANOSTRUCTURED} CARBOXYCELLULOSE FROM BIOMASS

Carboxycellulose nanofibers can be obtained through several approaches One of the most efficient approaches is the TEMPO-mediated oxidation

Biomass is a vast source of nanoscale cellulose, which can be chemically modified to act as an effective water purification material.

acetylation and silylation) introduces reaction, which converts $-\mathrm{OH}$ negative charges in the cellulose structure, groups in the cellulose polymer into which prom sites for the adsorntion of dissolved species. For example carboxycellulose whilst maintaining long fibre length nanofibers offer very large surface areas and chemically active functional groups, which make them ideally suited for filtration membranes and adsorption media for water treatment.

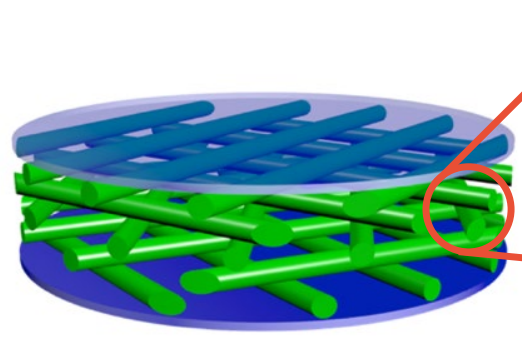

Plant cell wall

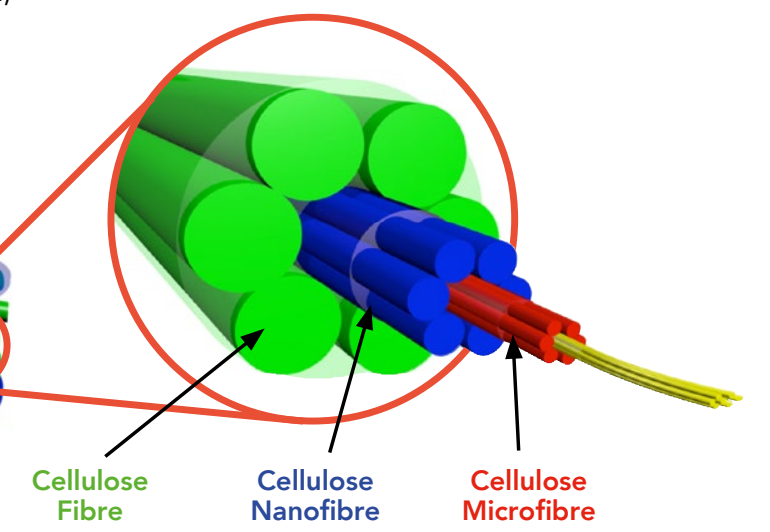

A hierarchical structure of cellulose fibres with different diameters in a plant cell wa matrix (through processes like oxidation, 
method is carried out as a sequence of several steps, and it requires speciality sodium bromide and TEMPO ronents) that generate dangerous radical species. Its sustainability as a large-scale process to produce nanostructured carboxycellulose remains, therefore, limited. Alternative approaches
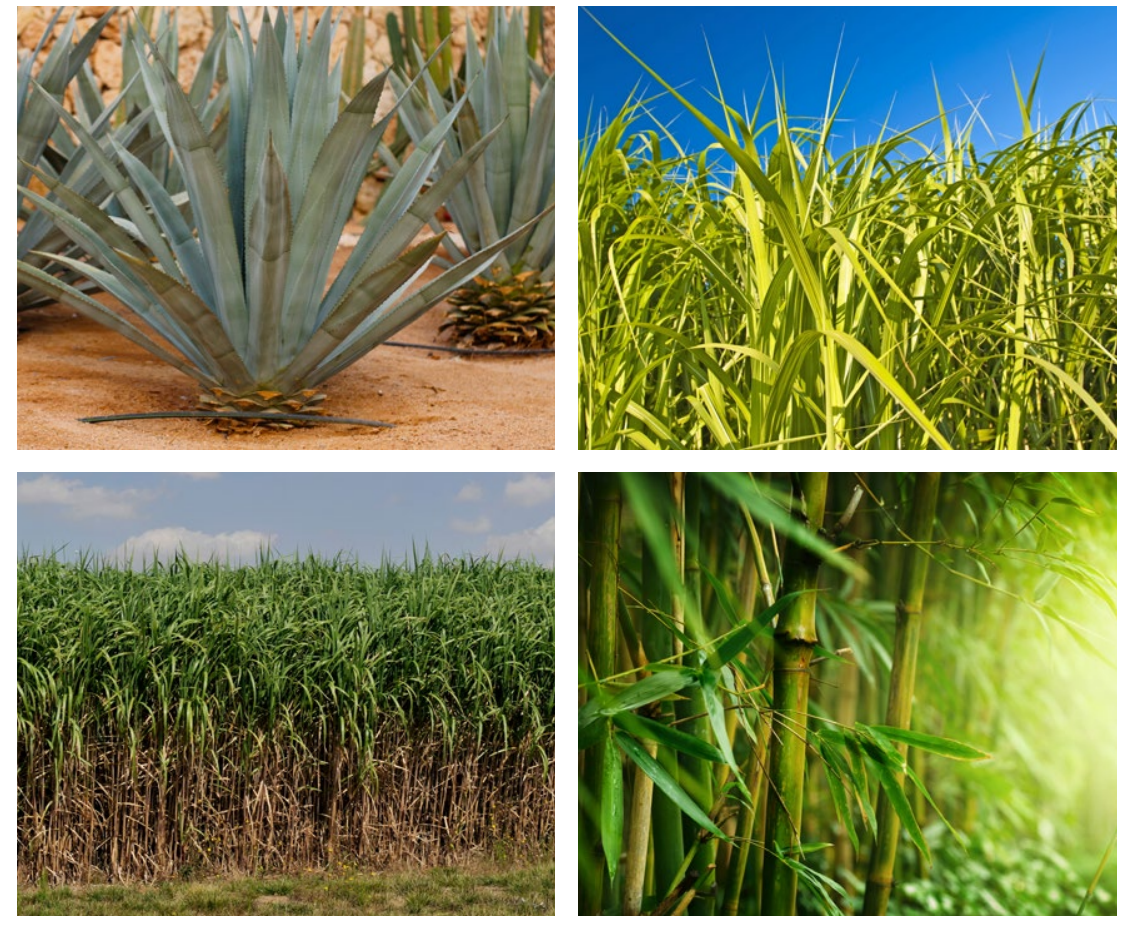

Carboxycellulose nanofibres obtained using the nitro-oxidation method exhibit very exceedingly high affinity for several common water contaminants.

of excess acid, which can attack hydroxyl Cellulose nanofibers obtained by nitrogroups in cellulose and produce carboxylate groups. The resulting materials for the removal of heavy metal ions, including lead, cadmium, mercury, higher fibre length and astatialy than those of cellulose nanoct ratio Furthermo the efluent obtained as a by-product can be neutralised efficiently using (inexpensive) sodium or ich salts that can be, to give nitrogenin agriculture.

\section{Behind the Research}

\section{Dr Benjamin S. Hsiao}

E: Benjamin.hsiao@stonybrook.edu T: +16318394402 W: www.hsiaoglobal.org have also been proposed, based on etherification, oxidation, esterification and carboxymethylation of cellulose, which are only effective for cellulose samples with small concentrations of lignin and hemicellulose and require preliminary treatment with chemicals like alkali and bleaching agents, along with mechanical treatment, to

NITRO-OXIDATION:

\section{A CLEAN ROUTE TO FUNCTIONAL} NANOCELLULOSE

Dr Hsiao and his co-workers have sustainable approach to the production of carboxycellulose nanofibers from untreated biomass, based on the use of a mixture of nitric acid (HNO) and sodium nitrite $\left(\mathrm{NaNO}_{2}\right)$ as the only required chemicals. This process has been shown to work very efficiently for untreated (raw) biomasses of various origins (such as jute, spinifex grass and bamboo cellulose) and, crucially, to be a strictly single-step treatment. This considerably reduces the electrical energy and the water consumption needs compared to other methods. It has been hypothesised that $\mathrm{HNO}_{3}$ in the of the untreated biomass by removing non-cellulosic components. The reaction of $\mathrm{HNO}_{3}$ and $\mathrm{NaNO}_{2}$ generates nitrosonium ions $\left(\mathrm{NO}^{+}\right)$in the presence

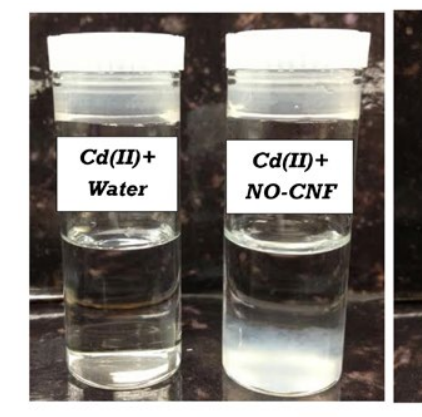

$2550 \mathrm{mg} / \mathrm{g}$

\section{Collaborators}

- Dr Priyanka R. Sharma as bacteria from water. For heavy me ons, adsorption capacilies several been reported. The metal adsorbed nanocellulose flocs can easily be removed using simple and inexpensive gravity driven microfiltration or decanting.

\section{IMPACT}

Vast amounts of biomass sources,

including agriculture waste, weeds cost, and all of them provide ideal raw materials for the nitro-oxidation process. The approach developed by Professor Hsiao represents a tremendous step forward toward the exploitation of underused resources like biomass to develop efficient processes for the removal of waterborne pathogens from countries and off-the-grid in developing Drunil K. Sharma<smiles>c1c#cccc#1</smiles>

Stony Brook University - The Claire Friedlander Family Foundation effective adsorbents in the most

Research Objectives

Dr Hsiao and his collaborators are focusing their research on the use of nanocellulose enabling

\section{Detail}

Chemistry Department

Stony Brook University

Bio

Dr Hsiao is a Distinguished Professor in Chemistry at Stony for Integrated Electric Energy Systems in Stony Brook University, with the mission to enhance the development of advanced technologies for the innovative nexus of food, nergy and water systems.

Funding

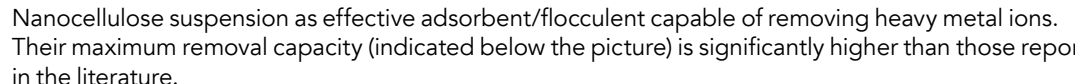

References

Hsiao, BS (2019). Benjamin S. Hsiao. [online]. Benjamin S. org/[Accessed 27 February 2019].

Sharma, PR; Chattopadhyay, A; Sharma, SK; Hsiao, BS

Carboxycellulose Nanofibers Prepared by the Nitro-Oxidation Method'. Industrial \& Engineering Chemistry Research, 56, 13885-13893.

Sharma, PR; Joshi, R; Sharma, SK; Hsiao, BS. (2017). 'A Simple Approach to Prepare Carboxycellulose Nanofibers from
Untreated Biomass'. Biomacromolecules, 18 (8), 2333-2341. Sharma, PR. Chattopadhyay, A. Zhan, C. Sharma, SK; Geng, L; Hsiao, BS. (2018). 'Lead removal from water using carboxycellulose nanofibers prepared by nitro-oxidation method. Cellulose, 25, 1961-1973.

Sharma, PR; Chattopadhyay, A; Sharma, SK; Amiralian, N; Martin, D; Hsiao, BS. (2018). 'Nanocellulose from Spinifex as an Effective Adsorbent to Remove Cadmium(II) From Water'

\section{Personal Response}

What are the key advantages of the nitro-oxidation method you have developed compared to existing agents, and what do you think are the most promising environments in which its application will have the largest impact?

II There are three key advantages of the nitro-oxidation method. First, the method greatly reduces the consumption of chemicals, energy and water. Second, the processing efluent can be efficaciously neutralised to produce plant fertilisers. Third, the method is effective to extract such as agriculture waste. The resulting nanocelllulose is proven to be an efficient water purification material (membrane or adsorbent) that can treat a wide range of water pollution problems. The demonstrated technology represents an innovative means to enhance the nexus far-reaching impacts to improve quality of life. 